Research Paper

\title{
Risk Factors for Pulmonary Complications Following Cardiac Surgery with Cardiopulmonary Bypass
}

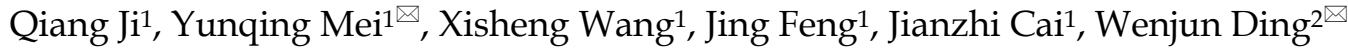 \\ 1. Department of Thoracic Cardiovascular Surgery of Tongji Hospital of Tongji University, Shanghai, P.R.China. 389 Xincun Rd., Shanghai, \\ 200065, P.R. China. \\ 2. Department of Cardiovascular Surgery of Zhongshan Hospital of Fudan University, Shanghai. 180 Fenglin Rd., Shanghai, 200032, \\ P.R.China.
}

\begin{abstract}
$\triangle$ Corresponding author: Dr Yunqing Mei, Department of Thoracic Cardiovascular Surgery of Tongji Hospital of Tongji University. 389 Xincun Road, Shanghai, 200065, P.R. China. E-mail: drjiqiang@tongji.edu.cn. Tel: (86)-21-66111073. Fax: (86)-21-56377580. Or Dr Wenjun Ding, Department of Cardiovascular Surgery of Zhongshan Hospital of Fudan University, Shanghai. 180 Fenglin Rd., Shanghai, 200032, P.R.China. E-mail: dwj12191@163.com. Tel: (86)-21-64041990. Fax: (86)-21-56050502.
\end{abstract}

(C) Ivyspring International Publisher. This is an open-access article distributed under the terms of the Creative Commons License (http:/ / creativecommons.org/ licenses/by-nc-nd/3.0/). Reproduction is permitted for personal, noncommercial use, provided that the article is in whole, unmodified, and properly cited.

Received: 2013.06.13; Accepted: 2013.08.26; Published: 2013.09.10

\begin{abstract}
Background: Pulmonary complications following cardiac surgery with cardiopulmonary bypass (CPB) are often associated with significant morbidity and mortality. However, few reports have focused on evaluating intra- and post-operative independent risk factors for pulmonary complications following cardiac surgery with CPB. This study aimed to evaluate peri-operative independent risk factors for postoperative pulmonary complications through investigating and analyzing 2056 adult patients undergoing cardiac surgery with CPB.

Methods: From lanuary 2005 to December 2012, the relevant pre-, intra-, and post-operative data of adult patients undergoing cardiac surgery with CPB in the department of cardiovascular surgery of Tongii Hospital of Tongii University in Shanghai were investigated and retrospectively analyzed. The independent risk factors for pulmonary complications following cardiac surgery with CPB were obtained through descriptive analysis and then logistic regression analysis.

Results: One hundred and forty-three adult patients suffered from pulmonary complications following cardiac surgery with CPB, with an incidence of $6.96 \%$. Through descriptive analysis and then logistic regression, independent risk factors for postoperative pulmonary complications were as follows: older age ( $>65$ years) $(\mathrm{OR}=3.3 \mathrm{I}, 95 \% \mathrm{Cl} \mathrm{I} .7 \mathrm{I}-7.13)$, preoperative congestive heart failure $(\mathrm{OR}=2.95,95 \% \mathrm{Cl}$ I.4I-5.84), preoperative arterial oxygenation $(\mathrm{PaO})(\mathrm{OR}=0.67,95 \% \mathrm{Cl}$ 0.33-0.85), duration of $\mathrm{CPB}(\mathrm{OR}=3.15,95 \% \mathrm{Cl} \mathrm{I} .55-6.2 \mathrm{I})$, intra-operative phrenic nerve injury $(\mathrm{OR}=4.59,95 \% \mathrm{Cl} 2.52-9.24)$, and postoperative acute kidney injury $(\mathrm{OR}=3.21,95 \% \mathrm{Cl} \mathrm{I} .9 \mathrm{I}-6.67)$. Postoperative pulmonary complication was not a risk factor for hospital death $(O R=2.10,95 \% \mathrm{Cl}$ 0.89-4.33).

Conclusions: A variety of peri-operative factors increased the incidence of pulmonary complications following cardiac surgery with cardiopulmonary bypass.
\end{abstract}

Key words: Pulmonary complication; Cardiac surgery; Cardiopulmonary bypass; Risk factor.

\section{Introduction}

Pulmonary complications are common complications following cardiac surgery with cardiopulmonary bypass (CPB) and are often associated with significant morbidity and mortality ${ }^{[1-5]}$. To clarify which patients are more prone to developing postoperative pulmonary complications may help to take effective measures to prevent, eliminate or ease postoperative pulmonary complications. Previous studies ${ }^{[6,7]}$ have 
reported the concept that postoperative pulmonary complications resulted from a combination of risk factors, mainly due to the nature of the patient population. However, few reports have focused on evaluating the intra- and post-operative risk factors for postoperative pulmonary complications ${ }^{[8]}$.

We hypothesized that besides the nature of the patient population, some intra- and post-operative parameters may be risk factors for postoperative pulmonary complications. This study reviewed 2056 adult patients undergoing cardiac surgery with $\mathrm{CPB}$ in the department of cardiovascular surgery of Tongji Hospital of Tongji University in Shanghai from January 2005 to December 2012 to evaluate pre-, intra-, and post-operative independent risk factors for postoperative pulmonary complications, in order to prevent, eliminate or ease postoperative pulmonary complications.

\section{Materials and methods}

\section{Definition}

Postoperative pulmonary complications included postoperative pneumonia and postoperative respiratory failure [9]. Postoperative pneumonia was defined as a positive result in a sputum culture requiring anti-infective treatment, or chest X-ray diagnosis of pneumonia following cardiac surgery. Postoperative respiratory failure was defined as the duration of mechanical ventilation more than 48 hours or re-intubation following cardiac surgery. Hospital mortality was defined as death that occurred during the same hospitalization or within 30 days of operation ${ }^{[10]}$.

Criteria for extubation included an alert and hemodynamically stable patient with no excessive bleeding, ability of the patient to breathe through a tracheal tube ( $\mathrm{T}$ tube) for at least 30 minutes with a fraction of inspired oxygen of less than 0.40 and a respiratory rate less than 25 breaths $/ \mathrm{min}, \mathrm{PaO}_{2}$ greater than $70 \mathrm{mmHg}$, a $\mathrm{PaCO}_{2}$ less than $40 \mathrm{mmHg}$ and a $\mathrm{pH}$ greater than 7.35 , with no metabolic acidosis. Other criteria included a tidal volume of $6 \mathrm{ml} / \mathrm{kg}$, a peak negative inspiratory pressure of less than $-20 \mathrm{cmH}_{2} \mathrm{O}$ and a mandatory chest radiograph before extubation to rule out pneumothorax, pleural effusion and atelectasis.

\section{Clinical data}

This study protocol was approved by the ethics committee of Tongji University and was consistent with the Declaration of Helsinki.

From January 2005 to December 2012, all adult patients undergoing cardiac surgery with $\mathrm{CPB}$ were investigated and retrospectively analyzed. Preopera- tive information included age, gender, body mass index, recent smoking (within 4 weeks of surgery), chronic obstructive pulmonary disease, hypertension, hypercholesterolemia, diabetes mellitus, cerebrovascular disease, chronic renal dysfunction (creatinine $>2.5 \mathrm{mg} / \mathrm{dL}$ or requiring dialysis), recent myocardial infarction (evidence of myocardial infarction within the last 30 days before surgery), preoperative heart failure (New York Heart Association class III and IV), preoperative vasoactive drugs support, atrial fibrillation, prior heart operation, impaired left ventricular function (left ventricular ejection fraction $<35 \%$ ), left ventricular end-diastolic diameter, long-term use of steroid hormone, $\mathrm{PaO}_{2}$, and preoperative hemoglobin level. Intraoperative variables included emergency operation, type of operation, and duration of CPB and aortic cross-clamping (ACC). Postoperative variables included peri-operative use of intra-aortic balloon pump (IABP), postoperative arrhythmia, acute myocardial infarction (new Q-wave infarction within 48 hours following surgery), acute kidney injury (absolute increase greater than $0.3 \mathrm{mg} / \mathrm{dl}$ or relative increase greater than $50 \%$ in the serum creatinine within $48 \mathrm{~h}$ after surgery, or requiring dialysis), postoperative cerebral vascular accident (new permanent neurologic event), pleural effusion, sternal infection (bone-related; any drainage of purulent material from the sternotomy wound, and instability of the sternum), reoperation for bleeding (re-operation to control bleeding within $36 \mathrm{~h}$ following initial surgery), anaemia (haematocrit less than 34\%), and right or bilateral phrenic nerve injury ${ }^{[11-14]}$. Right phrenic nerve injury was considered present when the dome of the right hemidiaphragm was two or more intercostal spaces higher than the left as seen on a plain chest roentgenogram or when right phrenic nerve transection was noted intraoperatively [15]. In addition, postoperative pneumonia, postoperative respiratory failure, re-intubation and duration of mechanical ventilation were recorded.

The primary endpoint was the occurrence of postoperative pulmonary complications comprising postoperative pneumonia and postoperative respiratory failure. The secondary endpoint was a composite of mortality and major adverse cardiac and cerebral vascular events (myocardial infarction, stroke, renal failure) occurring during the same hospitalization or within 30 days of operation.

\section{Anesthesia and cardiopulmonary bypass pro- cedure}

All patients underwent general anesthesia. Anesthesia was induced with midazolam (2-3mg), fentanyl $(0.2 \mathrm{mg})$, propofol $(0.5-1.5 \mathrm{mg} / \mathrm{kg})$ and vecuronium, and was maintained with inhaled isoflurane 
and continuous infusion of propofol (2 to 5 $\mathrm{mg} / \mathrm{kg} / \mathrm{h}$ ). 0.1-0.2mg fentanyl was intravenously administered before skin incision, sternotomy, aortic cannulation and initiation of cardiopulmonary bypass, respectively; and total amount of fentanyl was less than $15 \mu \mathrm{g} / \mathrm{kg}$ during operation.

All operations were performed with a midline incision and CPB. CPB was instituted with a single two-stage right atrial cannulation or double vena cava cannulation and an ascending aorta perfusion cannulation or femoral aortic perfusion cannulation. Standard management included membrane oxygenators, arterial catheter filters, and non-pulsatile flow of $2.4 \mathrm{~L} / \mathrm{min} / \mathrm{m}^{2}$, with a mean arterial blood pressure greater than $50 \mathrm{mmHg}$. Patients were heparinized with $300 \mathrm{IU} / \mathrm{kg}$ to achieve an activated clotting time $>480$ s. Heparin was neutralized with $1 \mathrm{mg}$ protamine sulfate per 100 IU given. Cold-blood cardioplegia and epicardial ice shavings (protective pads for phrenic nerve were not used) were used during cardiac surgery. Cold-blood cardioplegia was accomplished with anterograde delivery through the aortic root or coronary artery orifices and retrograde delivery through the coronary sinus.

\section{Statistical analysis}

Statistical analysis was performed using the SPSS13.0 statistical software package. All $p$ values $<0.05$ were considered to be statistically significant. Univariate analysis, using the unpaired $t$-test according to homogeneity test for variance to compare measurement data and Fisher's exact test to compare enumeration data, was performed to assess statistically significant variables, and those with $p<0.10$ were then entered into a logistic regression analysis to identify the independent risk factors for postoperative pulmonary complications. The regression coefficients of factors with $p<0.05$ were calculated. The Hosmer-Lemeshow goodness of fit coefficient was computed for the regression model. The stepwise multivariate logistic regression was also performed to assess the influence of postoperative pulmonary complications as an independent risk factor on hospital death.

\section{Results}

From January 2005 to December 2012, 2056 consecutive adult patients ( 585 females, with a mean age of $46.3 \pm 4.3$ years old of age) undergoing cardiac surgery with $\mathrm{CPB}$ were investigated and retrospectively analyzed. Among them, 143 patients, accounting for $6.96 \%$ of the total population, suffered from postoperative pulmonary complications (112 patients suffering from postoperative pneumonia, 64 patients suffering from postoperative respiratory failure, and
11 patients undergoing re-intubation) and were included in the PPC group (group of postoperative pulmonary complications), and the remaining patients (1913 patients) did not receive postoperative pneumonia or postoperative respiratory failure or re-intubation and were included in the non-PPC group. The PPC group had significantly longer duration of mechanical ventilation as compared to the non-PPC group ( $36.4 \pm 8.7$ hours versus $16.8 \pm 3.7$ hours, $\mathrm{p}<0.0001$ ).

Result of the univariate analysis is shown in Table 1 . It shows that older age, woman, recent smoking, chronic obstructive pulmonary disease, preoperative congestive heart failure, preoperative vasoactive drugs support, preoperative $\mathrm{PaO}_{2}$, duration of $\mathrm{CPB}$ and $A C C$, intra-operative phrenic nerve injury, peri-operative use of IABP, postoperative cerebral vascular accident, postoperative new onset of atrial fibrillation, postoperative acute kidney injury and postoperative anaemia were relative risk factors for postoperative pulmonary complications.

Those variables with $p<0.10$ obtained through the univariate analysis were then entered into multivariate logistic regression analysis (PPC or not as independent variable, variables with $p<0.10$ obtained through univariate analysis as dependent variables). As shown in Table 2, independent risk factors for postoperative pulmonary complications included older age ( $>65$ years) (OR=3.31, 95\% CI 1.71-7.13), preoperative congestive heart failure $(\mathrm{OR}=2.95$, $95 \% \mathrm{CI} 1.41-5.84)$, preoperative $\mathrm{PaO}_{2}(\mathrm{OR}=0.67,95 \% \mathrm{CI}$ 0.33-0.85), duration of $\mathrm{CPB} \quad(\mathrm{OR}=3.15$, 95\% CI 1.55-6.21), intra-operative phrenic nerve injury $(\mathrm{OR}=4.59,95 \% \mathrm{CI} 2.52-9.24)$, and postoperative acute kidney injury (OR=3.21, 95\% CI 1.91-6.67). The Hosmer-Lemeshow goodness of fit coefficient of this model was 0.90 .

In addition, 72 adult patients died within 30 days of cardiac surgery, with a hospital mortality of $3.5 \%$. The causes of death were as follows: low cardiac output $(n=36)$, sepsis $(n=24)$, malignant arrhythmia $(n=6)$, and gastrointestinal bleeding $(n=6)$. In logistic regression analysis, postoperative pulmonary complication was not a risk factor for hospital death (OR=2.10, 95\% CI 0.89-4.33).

\section{Discussions}

Pulmonary complications following cardiac surgery with cardiopulmonary bypass are common complications. Postoperative pulmonary complications manifest early as arterial hypoxemia, during the later course as pneumonia, and in rare cases also as acute lung injury. The incidence of postoperative pulmonary complications ranged from 3-16 percent following coronary artery bypass grafting and 5-7 
percent following cardiac valvular surgery $[2-5,7,9]$. In this study, 143 adult patients suffered from postoperative pulmonary complications, accounting for $6.96 \%$ of the total population. This result was consistent with previous reports $[2-5,7]$.

Table I. Comparison of clinical data between the two groups.

\begin{tabular}{|c|c|c|c|}
\hline Factors & $\begin{array}{l}\text { PPC } \\
(n=143)\end{array}$ & $\begin{array}{c}\text { Non-PPC } \\
(\mathrm{n}=1913)\end{array}$ & $\mathrm{p}$ value \\
\hline \multicolumn{4}{|l|}{ Pre-operation } \\
\hline Older age (over 65 years old) & $50(34.9 \%)$ & $215(11.2 \%)$ & $<0.0001$ \\
\hline Woman & $55(38.5 \%)$ & $530(27.7 \%)$ & 0.0071 \\
\hline $\operatorname{BMI}\left(\mathrm{kg} / \mathrm{m}^{2}\right)$ & $25.2 \pm 3.2$ & $24.9 \pm 2.5$ & 0.1757 \\
\hline Recent smoking & $57(39.9 \%)$ & $545(28.5 \%)$ & 0.0055 \\
\hline Diabetes mellitus & $20(13.9 \%)$ & $211(11.0 \%)$ & 0.2729 \\
\hline Hypertension & $43(30.1 \%)$ & $612(31.9 \%)$ & 0.7099 \\
\hline Hypercholesterolemia & $28(19.6 \%)$ & $405(21.2 \%)$ & 0.7498 \\
\hline COPD & $46(32.2 \%)$ & $114(5.9 \%)$ & $<0.0001$ \\
\hline Cerebrovascular disease & $18(12.6 \%)$ & $161(8.4 \%)$ & 0.0912 \\
\hline Long-term use of steroid hormone & $2(1.4 \%)$ & $6(0.3 \%)$ & 0.1020 \\
\hline Chronic renal dysfunction & $7(4.9 \%)$ & $55(2.9 \%)$ & 0.1968 \\
\hline Recent MI & $20(13.9 \%)$ & $186(9.7 \%)$ & 0.1113 \\
\hline Prior heart operation & $4(2.8 \%)$ & $21(1.1 \%)$ & 0.0907 \\
\hline Congestive heart failure & $52(36.4 \%)$ & $223(11.7 \%)$ & 0.0008 \\
\hline Hemoglobin level (mg/dl) & $11.0 \pm 2.2$ & $11.2 \pm 2.0$ & 0.2523 \\
\hline Impaired left ventricular function & $12(8.4 \%)$ & $102(5.3 \%)$ & 0.1285 \\
\hline Vasoactive drugs support & $22(15.4 \%)$ & $164(8.6 \%)$ & 0.0097 \\
\hline Atrial fibrillation & $49(34.3 \%)$ & $512(26.8 \%)$ & 0.0639 \\
\hline LVEDD (mm) & $52.9 \pm 7.6$ & $52.3 \pm 4.2$ & 0.1257 \\
\hline $\mathrm{PaO}_{2}(\mathrm{mmHg})$ & $74.8 \pm 8.3$ & $77.8 \pm 5.8$ & $<0.0001$ \\
\hline \multicolumn{4}{|l|}{ Intra-operation } \\
\hline Emergency surgery & $5(3.5 \%)$ & $38(2.0 \%)$ & 0.2186 \\
\hline Type of operation & & & 0.0732 \\
\hline Valve only & $65(45.4 \%)$ & $706(36.9 \%)$ & \\
\hline CABG only & $28(19.6 \%)$ & $305(15.9 \%)$ & \\
\hline Valve+CABG & $11(7.7 \%)$ & $73(3.8 \%)$ & \\
\hline Aortic surgery & $29(20.3 \%)$ & $61(3.2 \%)$ & \\
\hline $\mathrm{CHD}$ & $10(7.0 \%)$ & $768(40.2 \%)$ & \\
\hline $\mathrm{CPB}(\min )$ & $95.8 \pm 15.5$ & $92.5 \pm 12.6$ & 0.0030 \\
\hline $\mathrm{ACC}(\mathrm{min})$ & $78.6 \pm 8.8$ & $76.3 \pm 7.8$ & 0.0008 \\
\hline \multicolumn{4}{|l|}{ Post-operation (before extubation) } \\
\hline Use of IABP & $13(9.1 \%)$ & $72(3.8 \%)$ & 0.0067 \\
\hline AMI & $2(1.4 \%)$ & $6(0.3 \%)$ & 0.1020 \\
\hline New onset of atrial fibrillation & $39(27.3 \%)$ & $317(16.6 \%)$ & 0.0019 \\
\hline Pleural effusion & $21(14.7 \%)$ & $195(10.2 \%)$ & 0.1179 \\
\hline Acute kidney injury & $35(24.5 \%)$ & $187(9.8 \%)$ & $<0.0001$ \\
\hline Cerebral vascular accident & $12(8.4 \%)$ & $81(4.2 \%)$ & 0.0334 \\
\hline Sternal infection & $8(5.6 \%)$ & $62(3.2 \%)$ & 0.1464 \\
\hline Re-operation for bleeding & $5(3.5 \%)$ & $30(1.6 \%)$ & 0.0911 \\
\hline Anaemia & $46(32.2 \%)$ & $437(22.8 \%)$ & 0.0139 \\
\hline Phrenic nerve injury & $29(20.3 \%)$ & $95(5.0 \%)$ & $<0.0001$ \\
\hline
\end{tabular}

PPC: postoperative pulmonary complications; BMI: body mass index; COPD: chronic obstructive pulmonary disease; MI: myocardial infarction; LVEDD: left ventricular end-diastolic diameter; $\mathrm{PaO}_{2}$ : arterial oxygenation; $\mathrm{CABG}$, coronary artery bypass grafting; $\mathrm{CHD}$, congenital heart disease; $\mathrm{CPB}$ : cardiopulmonary bypass; $\mathrm{ACC}$ : aortic cross clamping; IABP, intra-aortic balloon pump; AMI: acute myocardial infarction. 
Table 2. Independent risk factors for PPC.

\begin{tabular}{llll}
\hline Factors & OR & $95 \% \mathrm{CI}$ & $\mathrm{p}$ value \\
\hline Older age (over 65 years old of age) & 3.31 & $1.71-7.13$ & 0.0035 \\
Preoperative congestive heart failure & 2.95 & $1.41-5.84$ & 0.0131 \\
Preoperative $\mathrm{PaO}_{2}$ & 0.67 & $0.33-0.85$ & 0.0245 \\
Duration of cardiopulmonary bypass & 3.15 & $1.55-6.21$ & 0.0171 \\
Intra-operative phrenic nerve injury & 4.59 & $2.52-9.24$ & 0.0046 \\
Postoperative acute kidney injury & 3.21 & $1.91-6.67$ & 0.0072 \\
\hline
\end{tabular}

The Hosmer-Lemeshow goodness of fit coefficient of this model was 0.90 . PPC, postoperative pulmonary complications; OR: odds ratio; $\mathrm{CI}$ : confidence interval; PaO 2 : arterial oxygenation.

It is reported that older age, smoking, obesity, diabetes, chronic obstructive pulmonary disease, hypercapnia, hypoproteinemia, prolonged duration of $\mathrm{CPB}$, postoperative bacteremia, postoperative endocarditis, postoperative gastrointestinal hemorrhage with or without perforation, postoperative acute kidney injury, sternal infection, postoperative cerebral vascular accident and reoperation for bleeding increased the incidence of postoperative pulmonary complications [16, 17]. This study revealed that independent risk factors for postoperative pulmonary complications included older age ( $>65$ years), preoperative congestive heart failure, low preoperative $\mathrm{PaO}_{2}$, prolonged cardiopulmonary bypass, intra-operative phrenic nerve injury, and postoperative acute kidney injury.

Older age was one of the major risk factors contributing to postoperative pulmonary complications. Patients aged more than 65 years undergoing cardiac surgery with CPB often had multiple concurrent diseases with poor cardiopulmonary reserve. They had a further reduction in their cardiopulmonary performance after cardiac surgery, and were more prone to postoperative low cardiac output and requiring longer ventilation support, and then were more prone to postoperative pulmonary complications.

Preoperative congestive heart failure was an important risk factor for postoperative pulmonary complications. Patients with preoperative congestive heart failure often had left atrial hypertension and pulmonary interstitial edema, which in turn resulted in a change in the pulmonary ventilation/blood flow ratio, thus causing postoperative hypoxemia and prolonged ventilation support [18, 19]. Preoperative congestive heart failure caused the lymph back to obstacle, pulmonary alveolar edema occurred then, which in turn could have resulted in a change in the pulmonary compliance, thus further aggravating respiratory dysfunction. Therefore, patients with preoperative congestive heart failure were prone to postoperative pulmonary complications. Accordingly, proper preoperative management of congestive heart failure in patients undergoing scheduled cardiac sur- gery with $\mathrm{CPB}$ might contribute to reduce the incidence of postoperative pulmonary complications.

Low preoperative $\mathrm{PaO}_{2}$ often suggested poor cardiopulmonary function or severe chronic obstructive pulmonary disease in patients. After cardiac surgery with $\mathrm{CPB}$, further reduction in cardiopulmonary function was expected in patients with low preoperative $\mathrm{PaO}_{2}$ who consequently were more prone to low cardiac output and requiring longer ventilation support, and thus were more prone to postoperative pulmonary complications. Therefore, proper peri-operative management of cardiopulmonary dysfunction might contribute to improve cardiopulmonary function and shorten postoperative ventilation support, and then reduce the incidence of postoperative pulmonary complications.

Prolonged cardiopulmonary bypass was also a major risk factor for postoperative pulmonary complications. Cardiopulmonary bypass is associated with a systemic inflammatory response, the production of oxygenderived free radicals, the activation of polymorphonuclear neutrophils, and the complement cascade and release of vessel constringent factors [20, 21]. All these have negative influences on important organs, such as the heart, lung, brain, and kidney. Prolonged cardiopulmonary bypass was expected to negatively influence cardiopulmonary and renal functions, and thus requirement of longer ventilation support and increase of postoperative pulmonary complications. In addition, prolonged cardiopulmonary bypass associated with complex disease, complicated operation, and imperfect myocardial protection may cause postoperative pulmonary complications. Thus, further refinement of surgical techniques and decrease in duration of cardiopulmonary bypass may contribute to reduction of postoperative pulmonary complications.

Phrenic nerve injury following cardiac surgery was variable in its incidence depending on the diligence with which it was sought. Definitive studies have shown this complication to be related to cold-induced injury during myocardial protection strategies and possibly to mechanical injury during 
internal mammary artery harvesting ${ }^{[22]}$. Right phrenic nerve injury, although rarely life-threatening, was more prone to lead to diaphragmatic paralysis, lung atelectasis, severe pulmonary dysfunction requiring prolonged mechanical ventilation or re-intubation, and other associated morbidities and even mortality. Right phrenic nerve injury was an independent risk factor for postoperative respiratory complications [22]. Although rare, bilateral phrenic nerve injury may lead to postoperative pulmonary complications [23].

Postoperative acute kidney injury, a common complication after cardiac surgery, remained an important adverse event and has been shown to be associated with postoperative complications, longer hospital stay, higher cost and increased mortality. Severe acute kidney injury, which usually combined with multiple organ dysfunction syndrome, low cardiac output, endotoxemia, hypervolemia, hyperkalemia and acidosis, requiring renal replacement therapy, had negative impact on respiratory function, causing prolonged ventilation support or re-intubation, and then, increasing the incidence of postoperative pulmonary complications [24].

Generally speaking, postoperative pulmonary complications led to increased mortality. In this study, postoperative pulmonary complication was not a risk factor for hospital death through multivariate logistic regression analysis. But further large multicenter studies are required.

The primary limitation of this study was its retrospective and observational nature, limiting generalization of its results. Another limitation of this study was the lack of detailed respiratory function data. $\mathrm{PaO}_{2}$ was measured preoperatively, but forced expiratory volume in 1 second $\left(\mathrm{FEV}_{1}\right)$ and forced vital capacity (FVC) were not routinely measured before cardiac surgery in the department of cardiovascular surgery of Tongji Hospital of Tongji University.

In conclusion, older age, preoperative congestive heart failure, low preoperative $\mathrm{PaO}_{2}$, prolonged cardiopulmonary bypass, intra-operative phrenic nerve injury, and postoperative acute kidney injury were six independent risk factors for pulmonary complications following cardiac surgery with cardiopulmonary bypass. Pulmonary complications following cardiac surgery with cardiopulmonary bypass may be reduced by shortening duration of cardiopulmonary bypass and avoiding intra-operative phrenic nerve injury. The proper peri-operative management of cardiopulmonary dysfunction may contribute towards reducing pulmonary complications following cardiac surgery with cardiopulmonary bypass and facilitate recovery.

\section{Competing Interests}

The authors have declared that no competing interest exists.

\section{References}

1. Canet J, Mazo V. Postoperative pulmonary complications. Minerva Anestesiol 2010; 76: 138-43.

2. Smetana GW. Schultz MJ. Pulmonary complications after cardiac surgery: ventilator associated lung injury? Med Sci Monit 2007; 13: LE3-4.

3. Rock P, Rich PB. Postoperative pulmonary complications. Curr Opin Anaesthesiol 2003; 16: 123-31.

4. Weissman C. Pulmonary complications after cardiac surgery. Semin Cardiothorac Vasc Anesth 2004; 8: 185-211.

5. Younossian AB, Adler D, Bridevaux PO, Kherad O. Postoperative pulmonary complications: how to anticipate and prevent the risk? Rev Med Suisse 2011; 7: 2214-9.

6. Silva DR, Gazzana MB, Knorst MM. Merit of preoperative clinical findings and functional pulmonary evaluation as predictors of postoperative pulmonary complications. Rev Assoc Med Bras 2010; 56: 551-7.

7. Smetana GW. Postoperative pulmonary complications: an update on risk assessment and reduction. Cleve Clin J Med 2009; 76: S60-5.

8. Canver CC, Chanda J. Intraoperative and postoperative risk factors for respiratory failure after coronary bypass. Ann Thorac Surg 2003; 759: 853-7.

9. Sachdev G, Napolitano LM. Postoperative pulmonary complications: pneumonia and acute respiratory failure. Surg Clin North Am 2012; 92: 321-44.

10. Arozullah AM, Henderson WG, Khuri SF, Daley J. Postoperative mortality and pulmonary complication rankings: how well do they correlate at the hospital level? Med Care 2003; 41: 979-91.

11. Amram JC, Michael GK, George F, Benjamin M, Diklah G, Arie S. Morbid results of prolonged intubation after coronary artery bypass surgery. Chest 2000; 118: 1724-31.

12. Filsoufi F, Rahmanian PB, Castillo JG, Mechanick JI, Sharma SK, Adams $\mathrm{DH}$. Diabetes is not a risk factor for hospital mortality following contemporary coronary artery bypass grafting. Interact Cardiovasc Thorac Surg 2007; 6: 753-8.

13. Antunes PE, Prieto D, Ferrão de Oliveira J, Antunes MJ. Renal dysfunction after myocardial revascularization. Eur J Cardiothorac Surg 2004; 25 : 597-604.

14. Ji Q, Mei Y, Wang X, et al. Study on the risk factors of postoperative hypoxemia in patients undergoing coronary artery bypass grafting. Circ J 2008; 72: 1975-80.

15. Deng Y, Byth K, Paterson HS. Phrenic nerve injury associated with high free right internal mammary artery harvesting. Ann Thorac Surg 2003; 76: 459-63.

16. Jensen L, Yang L. Risk factors for postoperative pulmonary complications in coronary artery bypass graft surgery patients. Eur J Cardiovasc Nurs 2007; 6: 241-6.

17. Lauruschkat AH, Arnrich B, Albert AA, Walter JA, Amann B, Rosendahl UP, Alexander T, Ennker J. Diabetes mellitus as a risk factor for pulmonary complications after coronary bypass surgery. J Thorac Cardiovasc Surg 2008; 135: 1047-53.

18. Andreassen S, Rees SE, Kjaergaard S, et al. Hypoxemia after coronary bypass surgery modeled by resistance to oxygen diffusion. Crit Care Med 1999; 27: 2445-53.

19. Kjaergaard S, Rees SE, Grønlund J, et al. Hypoxaemia after cardiac surgery: clinical application of a model of pulmonary gas exchange. Eur J Anaesthesiol 2004; 21: 296-301.

20. Lagan AL, Melley DD, Evans TW, Quinlan GJ. Pathogenesis of the systemic inflammatory syndrome and acute lung injury: role of iron mobilization and decompartmentalization. Am J Physiol Lung Cell Mol Physiol 2008; 294: L161-74.

21. Levy JH, Tanaka KA. Inflammatory response to cardiopulmonary bypass. Ann Thorac Surg 2003; 75: S715-20.

22. Tripp HF, Bolton JW. Phrenic nerve injury following cardiac surgery: a review. J Card Surg 1998; 13: 218-23.

23. Kohorst WR, Schonfeld SA, Altman M. Bilateral diaphragmatic paralysis following topical cardiac hypothermia. Chest 1984; 85: 65-8.

24. Faubel S. Pulmonary complications after acute kidney injury. Adv Chronic Kidney Dis 2008; 15: 284-96. 